\title{
Genomic organization of eukaryotic tRNAs
}

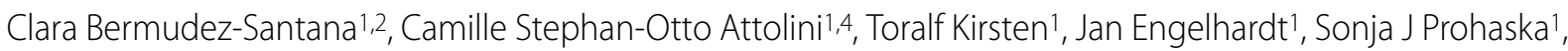 \\ Stephan Steigele ${ }^{3}$ and Peter F Stadler ${ }^{* 1,5,6,7,8}$
}

\begin{abstract}
Background: Surprisingly little is known about the organization and distribution of tRNA genes and tRNA-related sequences on a genome-wide scale. While tRNA gene complements are usually reported in passing as part of genome annotation efforts, and peculiar features such as the tandem arrangements of tRNA gene in Entamoeba histolytica have been described in some detail, systematic comparative studies are rare and mostly restricted to bacteria. We therefore set out to survey the genomic arrangement of tRNA genes and pseudogenes in a wide range of eukaryotes to identify common patterns and taxon-specific peculiarities.
\end{abstract}

Results: In line with previous reports, we find that tRNA complements evolve rapidly and tRNA gene and pseudogene locations are subject to rapid turnover. At phylum level, the distributions of the number of tRNA genes and pseudogenes numbers are very broad, with standard deviations on the order of the mean. Even among closely related species we observe dramatic changes in local organization. For instance, $65 \%$ and $87 \%$ of the tRNA genes and pseudogenes are located in genomic clusters in zebrafish and stickleback, resp., while such arrangements are relatively rare in the other three sequenced teleost fish genomes. Among basal metazoa, Trichoplax adhaerens has hardly any duplicated tRNA gene, while the sea anemone Nematostella vectensis boasts more than 17000 tRNA genes and pseudogenes. Dramatic variations are observed even within the eutherian mammals. Higher primates, for instance, have $616 \pm 120$ tRNA genes and pseudogenes of which $17 \%$ to $36 \%$ are arranged in clusters, while the genome of the bushbaby Otolemur garnetti has 45225 tRNA genes and pseudogenes of which only $5.6 \%$ appear in clusters. In contrast, the distribution is surprisingly uniform across plant genomes. Consistent with this variability, syntenic conservation of tRNA genes and pseudogenes is also poor in general, with turn-over rates comparable to those of unconstrained sequence elements. Despite this large variation in abundance in Eukarya we observe a significant correlation between the number of tRNA genes, tRNA pseudogenes, and genome size.

Conclusions: The genomic organization of tRNA genes and pseudogenes shows complex lineage-specific patterns characterized by an extensive variability that is in striking contrast to the extreme levels of sequence-conservation of the tRNAs themselves. The comprehensive analysis of the genomic organization of tRNA genes and pseudogenes in Eukarya provides a basis for further studies into the interplay of tRNA gene arrangements and genome organization in general.

\section{Background}

Transfer RNAs (tRNAs) are among the most ancient genes. They can be traced back to the putative RNA World [1] before the separation of the three Domains of Life. There is clear evidence, furthermore, that all tRNA gene are homologs, deriving from an ancestral " proto-

\footnotetext{
*Correspondence: studla@bioinf.uni-leipzig.de

1 Bioinformatics Group, Department of Computer Science and Interdisciplinary Center for Bioinformatics, University of Leipzig, Härtelstraße 16-18, D-04107, Leipzig, Germany

Full list of author information is available at the end of the article
}

tRNA" [2], which in turn may have emerged from even smaller components, see e.g. [3-7].

Besides their primary ancestral function in translation, tRNAs appear to have acquired several additional modes of employment throughout evolution. Several recent studies, for instance, reported tRNA-derived small RNAs in different Eukaryotic clades [8-12], which at least in part appear to be utilized in the RNAi pathway. Furthermore, tRNA genes are a prolific source of repetitive elements (SINEs) [13], and of tRNA-derived small RNAs such as the small brain-specific non-messenger RNA BC1 RNA $[14,15]$ and other SINE-derived ncRNAs [16]. 
Multiple copies of functional tRNA genes, the existence of numerous pseudo-genes and tRNA-derived repeats are general characteristics of tRNA evolution throughout Eukarya [17]. In general, tRNA genes appear to evolve rapidly. In E. coli, the rate of tRNA gene duplication/deletion events is of the order of one per million years [18], and a recent analysis of schistosome genomes revealed striking differences in the tRNA complement between the close related platyhelminths $S$. mansoni and S. japonicum [19].

Although the tRNAs themselves and their sequence and structural evolution has received quite a bit of attention [20-23], much less is known about the genomic organization of tRNA genes. Recent evidence, however, indicates that tRNA genes play a role in eukaryotic genome organization [24] e.g. by acting as barriers that separate chromatin domains. In trypanosomes, for example, tRNA genes mostly appear at the boundaries of transcriptional units and may be involved in the deposition of special nucleosome variants in these regions [25]. Furthermore, there is a link between tRNA loci, in particular clusters of tRNA genes, and chromosomal instability [2630]. A recent study showed that tRNA genes may act as barriers to DNA replication fork progression [24], providing a possible mechanism for the formation of genomic fragile sites. The genomic evolution of tRNA gene thus may be linked to the evolution of genome organization. Nevertheless, reports on clade-specific features, such as the strong increase of tRNA introns in Thermoproteales [31], are rare.

A peculiar feature of tRNA gene organization are tRNA tandem repeats, which so far have been reported only in the protistan parasite Entamoeba histolytica [32,33]. MicroRNAs derived from a precursor in which an imperfectly matched inverted repeat forms a partly doublestranded region, as observed in Chlamydomonas [34,35], furthermore, suggests that head-to-head or tail-to-tail arrangements of tRNA gene might form an evolutionary source of small RNAs.

In this contribution, we survey the genomic distribution of tRNA genes and pseudogenes throughout the Eukarya and provide a comprehensive comparative view of the eukaryotic tRNA genomics. Our study makes use of the near-perfect sensitivity and specificity of tRNAscan-SE[36], which reliably determines the complete tRNA complement of eukaryotic genomes.

\section{Results and Discussion Numbers of tDNAs}

For each of the 74 genomes included in our survey we collected summary statistics on the number of tRNA gene and tRNA pseudogenes as well as on their genomic clusters. To simplify the language, we will use the term " tDNA" to refer to both tRNA genes and tRNA pseudo- genes, while " tRNA gene" will be reserved to loci with probably intact tRNA sequences. In practise, we use tRNAscan-SE to distinguish between tRNA genes and tRNA pseudogenes (see Methods for details).

We define two adjacent tRNA gene or tDNAs as " clustered" if their distance is less than 1000 nucleotides. This threshold is motivated by a statistical analysis of the distances between adjacent tDNA loci, which shows that at this distance we have to expect very few or no tDNA pairs in the genomes under investigation (see Methods for details). We then distinguish between homogeneous clusters, consisting of tDNA with the same isoacceptor family (i.e., coding for the same aminoacid), and heterogeneous clusters. Within clusters, we separately consider the three relative orientations $\rightarrow \rightarrow, \leftarrow \rightarrow$, and $\rightarrow \leftarrow$. Data have been analyzed for putatively functional tRNA gene (as classified by tRNAscan-SE), and for all tDNAs. Fig. 1 shows a sample of a graphical representation of the survey results. The full figure comprising all 74 genomes is provided as Additional File 1. Complete lists of tDNAs in gff format can be found at the website [37].

Despite an overall correlation with genome size, there does not seem to be a general trend in the number of tRNA genes. Although some mammals, for instance, exhibit tens or even hundreds of thousands of tDNA copy numbers, other mammalian genomes harbour only a few hundred copies. For instance, old world monkeys and great apes have about $616 \pm 120$ tDNAs, while the related bushbaby (Otolemur garnetti) exhibits 45225 tDNAs. The highest counts are reached for the cow and rat genomes with more than 100000 tDNAs. For the 12 sequenced Drosophila species, we find $320 \pm 73$ tDNAs. Trichoplax adhaerans, one of the most basal animals has no more than 50 tRNA genes, while the cnidaria Nematostella vectensis has more than 17000 . Within teleosts, tDNAs range from about 700 in Tetraodontiformes to 20000 in zebrafish. Variations by about an order of magnitude are also common in other major clades. Naegleria gruberi, for example has 924 tDNAs, while Kinetoplastids (Leishmania and Trypanosoma have only 91 and 65 copies). Surprisingly, the variation is very small in the "green lineage". Spermatophyta show little variation with $706 \pm$ 96 loci, the basal land plants Physcomitrella patens (432 tDNAs) and Selaginella moellendorffii (1290 tDNAs) and even the unicellular algae Volvox carteri (1051 tDNAs) and Chlamydomonas reinhardtii (336 tDNAs) have similar numbers.

Despite the often large variation among even closely related lineages, we observe the expected correlation between the number of tDNAs with genome size, Fig. 2. The correlation is significant, with correlation coefficient $\rho \in(0.71 \ldots 0.76)$, but subject to a high level of variation reflecting large differences in the evolutionary history of different lineages. While the total number of tDNAs 


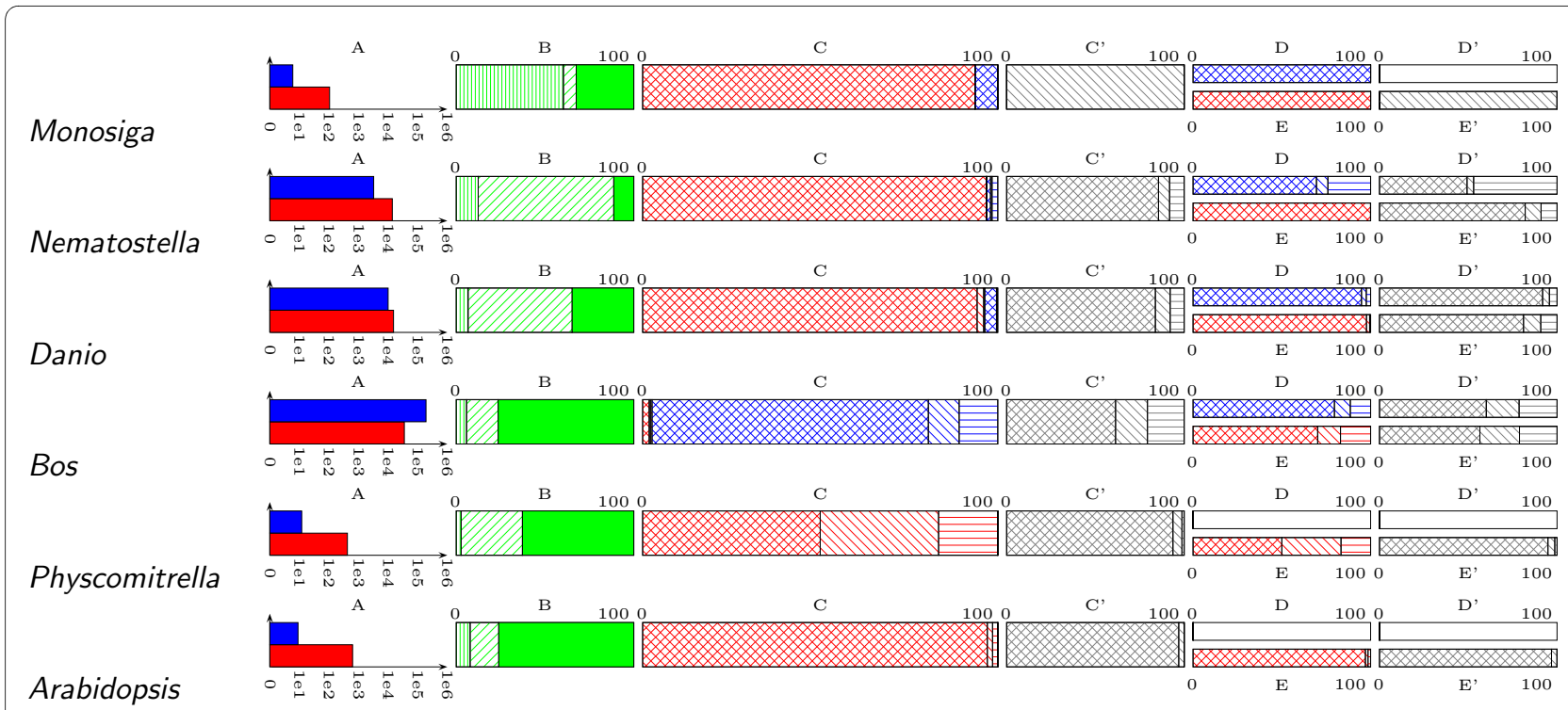

Legend:

A Distribution of tRNA genes and tRNA pseudogenes $\square$ on a logarthmic scale

B Fraction of tDNAs located in:

genomic clusters III homogeneous clusters // heterogeneous clusters not located in clusters.

C Fraction of homogeneous pairs:

pairs tRNA genes $\mathrm{XX} \rightarrow \rightarrow, \backslash \backslash \rightarrow \leftarrow, \equiv \leftarrow \quad$ pairs tRNA pseudogenes $\mathrm{XX} \rightarrow \rightarrow, \backslash \backslash \rightarrow \leftarrow, \equiv \leftarrow$

C' Fraction of Heterogeneous pairs: $\mathrm{XX} \rightarrow \rightarrow, \backslash \backslash \rightarrow \leftarrow, \equiv \leftarrow \rightarrow$

D, D' analogous to C, C' separately for tRNA genes

E, E' analogous to $C^{\prime}, C^{\prime}$ separately for tRNA pseudogenes

Figure 1 Summary of tRNA gene and tDNA statistics

scales approximately linearly with genome size, $\alpha=0.93 \pm$ 0.10 , the growth in the number of intact, probably functional tRNA genes is much slower, consistent with $N^{2 / 3}$. The number of tRNA pseudogenes, on the other hand, grows faster than linearly, $\sim N^{1.61 \pm 0.18}$. The reasons for this difference in scaling remains unclear. One may speculate that selective forces maintain only a limited number of functional tDNA copies causing the sub-linear growth of intact tRNA genes with genome size, while the duplication/deletion mechanism acts towards a uniform coverage of the genome with a rate that is to a first approximation constant throughout eukaryotic genome, accounting for the linear growth of the total number of tDNAs.

Several selective forces could act on the tRNA genes and/or all tDNA loci to cap their number. The bias towards small deletions over insertions observed in [38] is one potential candidate that is independent of special properties of tRNAs. Variations in codon usage might provide another selection-based explanation for the variation of tDNA copy numbers. In eubacteria, a correlation between tRNA abundance, tRNA gene copy number, and codon usage is well established [39]. Whether codon bias causes tDNA copy number variation or vice versa remains topic of an intense debate, however. A mechanistic explanation describing the coevolution of codon usage with tRNA gene content is given in [40]. It remains unclear to what extent the correlation of tRNA copy numbers and codon usage carries over to eukaryotic genomes. A detailed investigation in Schistosoma mansoni and Schistosoma japonicum finds no correlation between tRNA gene numbers and codon usage, while a statistically significant but still very weak correlation is observed in Schmidtea mediterranea [19]. In Nasonia, the correlation of codon usage and the copy numbers of tRNA genes appears to be restricted to highly expressed genes. The strength of this correlation decreases with GC-content in plant genomes [41].

In any case, codon usage cannot be employed to explain the observed differences in tDNA copy numbers that span several orders of magnitude. These huge fluctuations, which are observed both within some lineages and between closely related lineages, argues against a mechanism that relies on selection on the tRNAs. Instead, the more than linear scaling of tRNA pseudogenes with genome size suggests a faster tDNA turnover in larger 


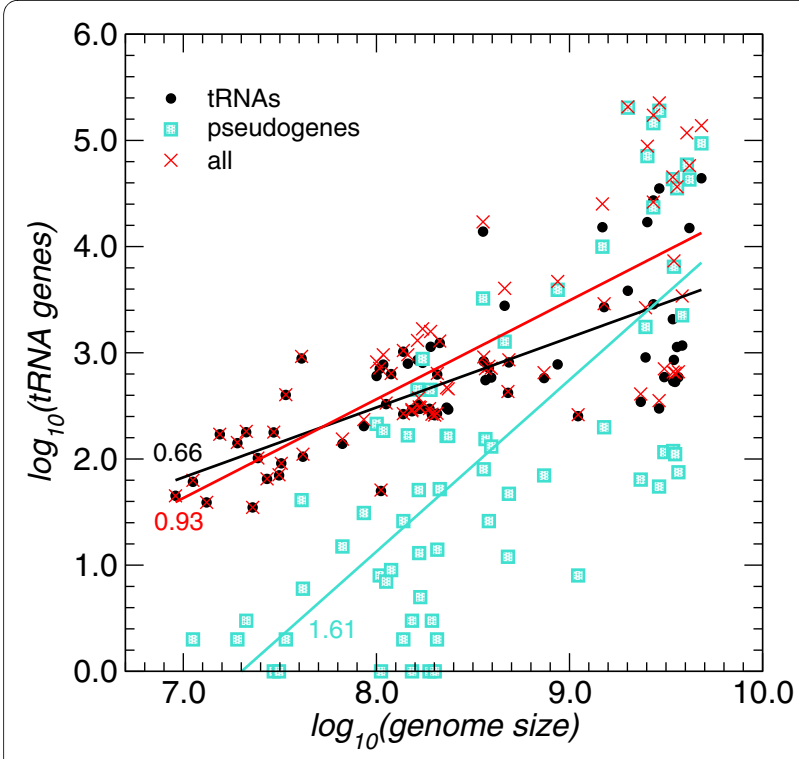

Figure $\mathbf{2}$ Correlation of the number of tDNAs with genome size The slopes of the three regressions are significantly different: Intact tRNA gene $(\cdot, a=0.658 \pm 0.076)$, tRNA pseudogenes $(\square, a=1.615 \pm$ $0.181)$, total number of tDNAs $(x, a=0.930 \pm 0.096)$.

genomes - after all, pseudogenes and gene relics are steps in the evolutionary degradation of genes.

\section{tDNA clusters}

In order to investigate the propensity for the formation of tDNA clusters, we consider the cumulative distribution of consecutive tDNA pairs as a function of their genomic distance. Based on a statistical evaluation of the distances between adjacent tDNAs (see Methods), we define two tDNAs to be clustered in the genome if they are located within 1000 nt.

Not surprisingly, in species with small tDNA copy number, clusters typically are rare. In Trichoplax adherens, for instance, all tDNAs are isolated. There is no clear-cut relation between tDNA copy number and clustering, however. In Nematostella vectensis $89 \%$ of the tDNAs appear in clusters. In mammals, which have even larger tDNA copy numbers, less than a quarter of the tDNAs appear in clusters. Again, there do not appear to be any large-scale phylogenetic regularities. In teleost fishes, for example, the stickleback Gasterosteus aculeatus has $87 \%$ clustered tDNAs, in zebrafish this number reaches $65 \%$. On the other hand, pufferfishes and medaka (Oryzias latipes) have predominantly isolated tDNAs. Similarly, large variation appears in other clades, see Fig. 1 and Additional File 2. Higher primates have 17\% to 36\% of their tDNAs in clusters, with the exception of the bushbaby Otolemur garnetii, with only $5.6 \%$ of its 45225 tDNAs located in clusters. In plants there are also no clear regularities. The fraction of clustered tDNAs stays below $25 \%$ in Spermatophyta, while the chlorophyceae
Volvox carteri and Chlamydomonas reinhardtii have $41 \%$ and $56 \%$ of their tDNAs localized in genomic clusters.

Most tDNA clusters are small, containing only a few colocalized tRNA genes. Typically, the frequency of larger clusters quickly decreases, at least approximately following an exponential distribution. This is particularly obvious in the case of mammals and drosophilids. In some cases, however, longer clusters are more abundant. Exceptionally large tDNA gene clusters, with fifty and more members, are observed for example in Nematostella and in the genomes of teleost fishes, Fig. 3.

The internal structure of tDNA clusters also differs widely between lineages. Fig. 1 and Additional Files 2 and 3 summarize the relative abundances of homogeneous and heterogeneous clusters, respectively. More precisely, we record the fraction of adjacent tDNA pairs coding for the same aminoacid. While Tetrahymena, Monosiga, and the drosophilids exhibit mostly homogeneous pairs, we observe mostly heterogeneous pairs in kinetoplastids, Nematostella, clawed frog, and zebrafish, see Fig. 4 for an example. In order to further investigate the structure of heterogeneous clusters we determined how often combinations two isoacceptor families appear in adjacent pairs. These data are conveniently represented in triangular matrices such as those in Fig. 5. Homogeneous clusters populate the main diagonal, whereas heterogeneous pairs are represented by off-diagonal entries. As for other features of the genomic tRNA distribution there are neither strong common patterns among all organisms investigated, nor are there systematic phylogenetic patterns. While Monosiga, for example, has almost exclusively homogeneous pairs, other species exhibit a wide variety of heterogeneous pairs. In Danio, for instance, K-N, K-S, and $\mathrm{R}-\mathrm{T}$ are most frequent. In the cow genome, many clusters involve tRNA pseudogenes, which are much less prevalent in the other three examples. In the cow, C-C pseudogenes account for more than $30 \%$ of the pairs. A comprehensive collection of co-occurrence tables is provided as Additional File 3. Not surprisingly, there is a general trend towards more complex co-occurrence matrices for species with larger numbers of tDNAs. Most adjacent tDNA pairs in both homogeneous and heterogeneous clusters have parallel orientation. If the arrangements were random, we would expect that $50 \%$ of pairs are of this type. In many cases, e.g. Arabidopsis, Selaginella, Xenopus, or Danio, nearly all pairs are in parallel. Among the anti-parallel pairs, some species have a strong bias for either head-to-head (e.g. primates, and Cryptococcus) or tail-to-tail arrangements (Oryza and Caenorhabiditis). Even within primates, the ratio of head-to-head and tailto-tail pairs varies considerably.

In most species with very large tDNA copy numbers we can expect some tDNA clusters to appear by chance. We tested this by randomizing the tDNA locations (see 

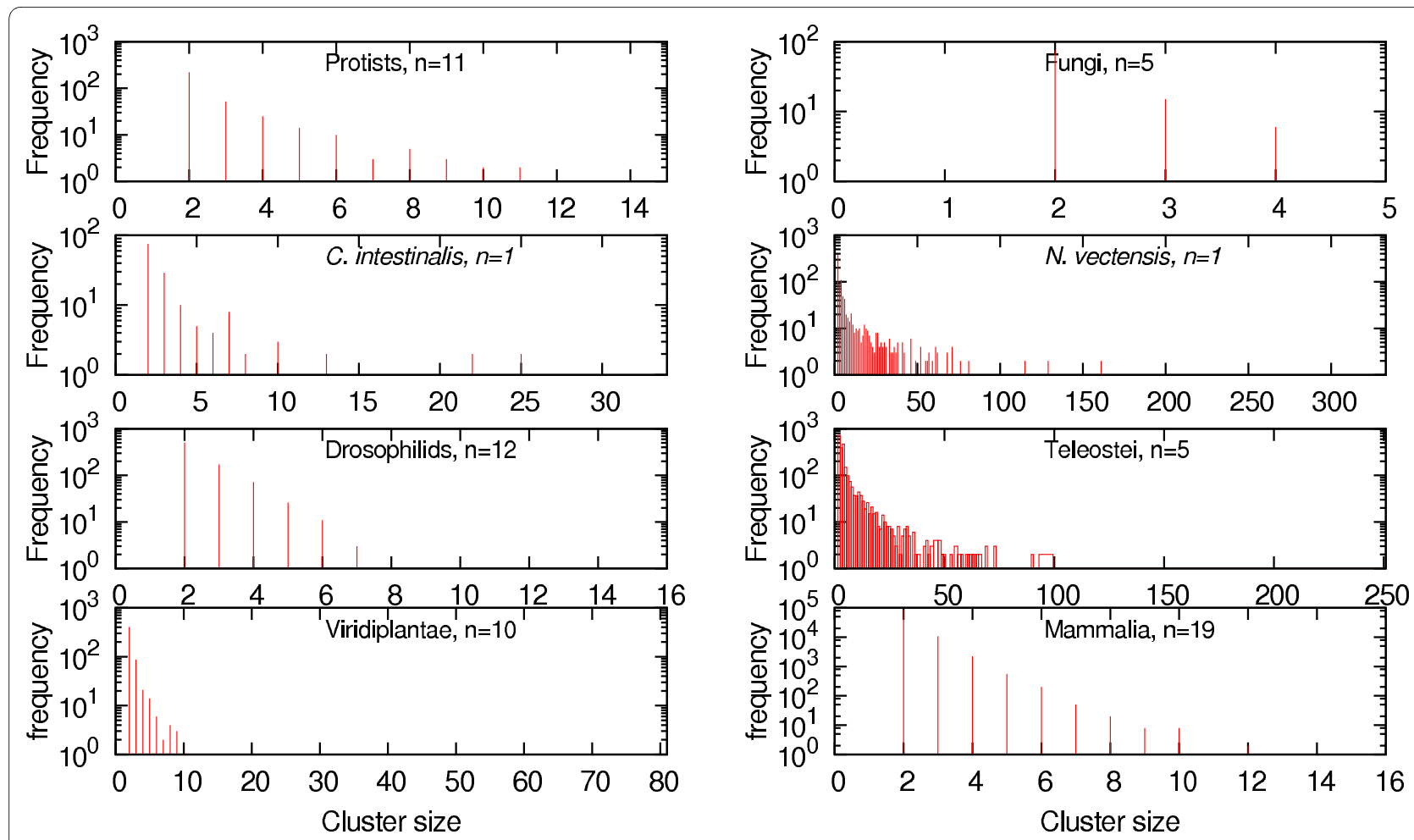

Figure 3 Distribution of tDNA clusters sizes for several lineages for which multiple sequenced genomes are available as well as some examples of individual genomes. Most tDNA clusters are small, and the frequency of long clusters rapidly decreases.

Methods for details). The results for eutherian mammals are compiled in Tab. 1, a full list of random pair configuration is given in Additional File 2. In most genomes, there are significantly more tDNA pairs than expected, suggesting a mode of tDNA evolution of favours the formation of local clusters. Local DNA duplications, also underlying the copy number variations within many populations (see e.g. $[42,43]$ and the references therein), are of course the prime suspects.

We observe significant under-representations of tDNA pairs only in a few species with very high tDNA counts: Dasypus novemcinctus, Felis catus, and Loxodonta afri- cana. At present, we have no biological explanation for this observation.

Clusters of tDNAs have been implicated in interfering with the DNA replication forks [26]. The tDNA clusters might thus be instrumental in orchestrating the timing of DNA replication. On the other hand, replication fork pause sites are associated with genomic instability [27-30] and hence may contribute to the rapid evolution of these tDNA clusters. Furthermore, retrotransposable elements tend to select tRNA genes as chromosomal integration sites [44], appearently in order to avoid gene disruptions upon retrotransposition. A recent comparison of yeast genomes associated genomic rearrengements, losses, and

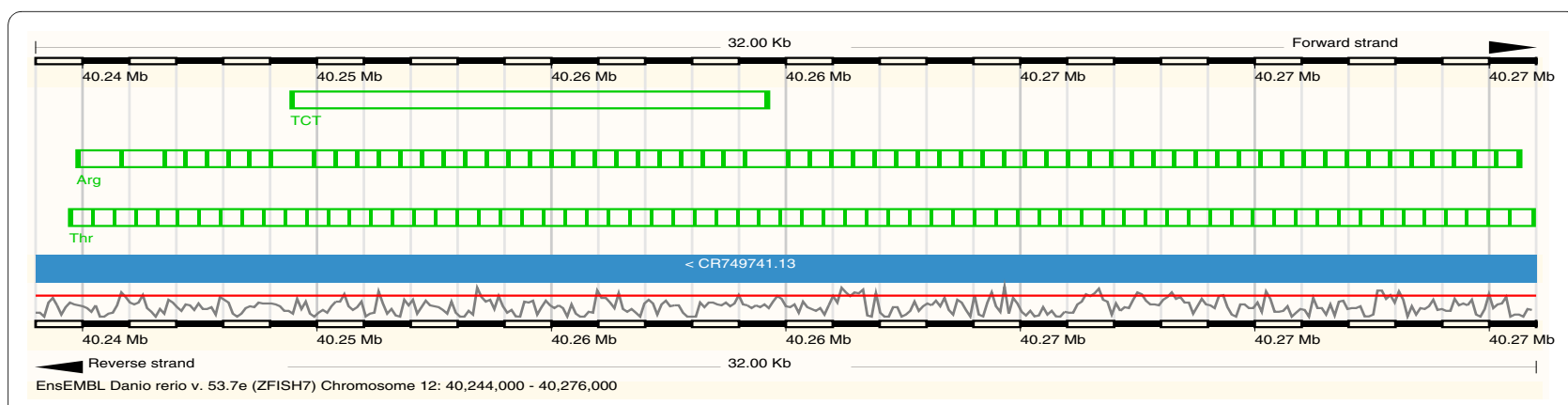

Figure 4 Example of heterogeneous tDNA cluster consisting of multiple copies of tRNA-Arg(TCT) and tRNA-Thr(AGT or TGT). Two tRNA pseudogenes with anti-codon TCT are interspersed. 


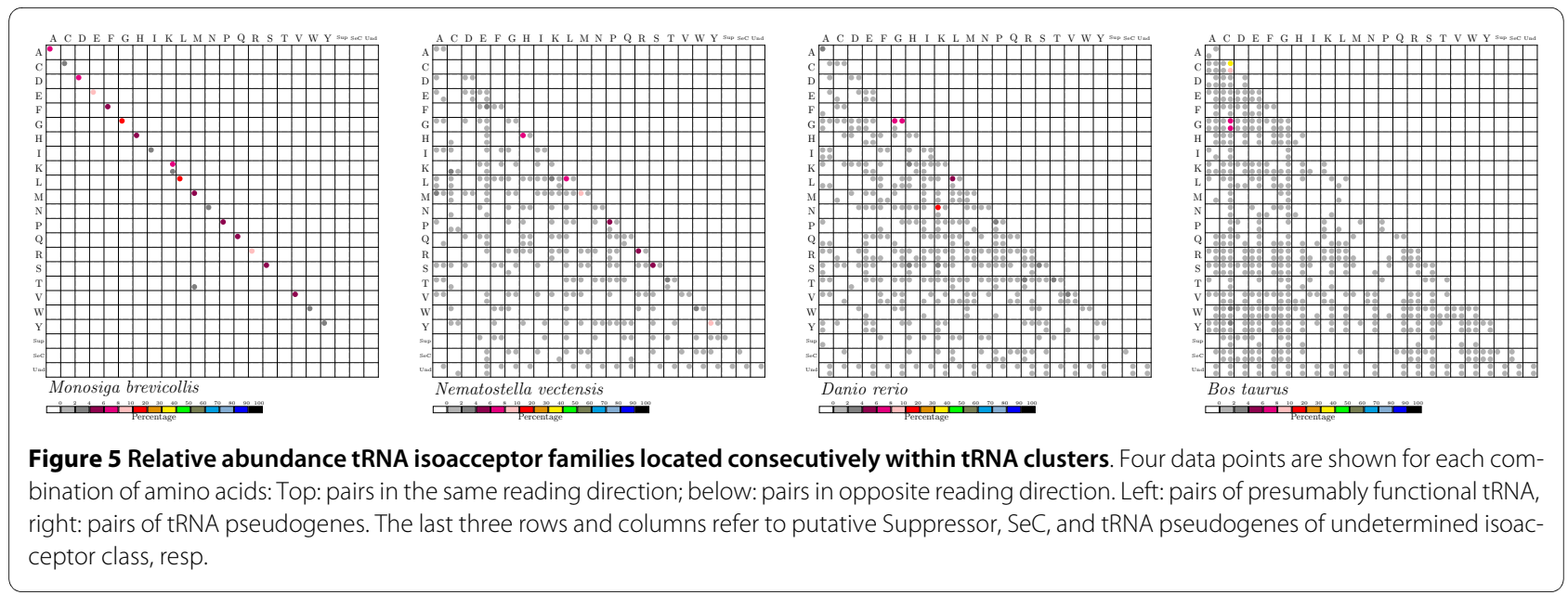

additions with tRNA genes [45]. Taken together, tDNA clusters thus appear as highly dynamic unstable genomic regions.

\section{Synteny}

Transfer RNAs have been reported to behave similar to repetitive elements as far as their genomic mobility is concerned. They appear to evolve via a rapid duplicationdeletion mechanism that ensures that copies of tRNA genes within a genome are usually more similar to each other than tRNA gene of different species $[18,46]$. In $E$. coli, for example, the rate of tRNA gene duplication/deletion events has been estimated to be about one event every 1.5 million years [18]. We are not aware of (semi)quantitative estimates from eukaryotes. Our analysis is consistent with this mechanism (see below).

Since tRNA genes with the same anticodon are typically nearly identical, the only way to estimate rates of tRNA gene turnover is to determine, for each tRNA-bearing locus, whether tDNAs can be found in a syntenic locations in evolutionarily related species. We have determined such data here for eight selected species, including six mammals, namely the Catarrihni Homo sapiens, Pan troglodytes, Pongo pygmaeus, and Macaca mulatta, the rodent Mus musculus, and the Marsupialia Monodelphis domestica. The data set includes also more distant vertebrates Gallus gallus and Xenopus tropicalis to investigate whether there are tDNAs with very stable genomic locations.

Tab. 2 shows the results for the one- and two-side linkage analysis (see Section Methods). The number of related synteny regions based on the single-side linkage analysis is significantly higher than the region number created by the two-side linkage analysis. Since the latter analysis approach is more restrictive, the results between both analysis approaches also differ. While synteny regions in related species are mostly assigned by the single-side linkage analysis, the results of the two-side link- age analysis are more differentiated. Therefore, we discuss only the results of two-side linkage analysis in the following.

Within Catarrhini, tDNA locations are quite well conserved. For instance $80 \%(394 / 493)$ of human tDNA regions are conserved in the chimp, and there are still $63 \%(284 / 450)$ of the rhesus tDNA locations recovered in chimp. Somewhat surprisingly, there is also a large fraction of syntenic loci between mouse and opossum $(80 \%$ $[19,466 / 24,352]$ of the mouse loci and $76 \%[16,634$ of $21,810]$ of the opossum loci). We suspect that the large fraction is confounded by the large overall number of tDNA loci and the rather larger intervals of five flanking genes used to define synteny, which taken together cover a substantial fraction of the genome. A second group of comparisons identified only a small number of syntenically conserved loci. Asymmetric results, which large retention in one direction is observed when the tDNA numbers are dramatically different. This concerns the comparisons between Catharrini, on the one hand, and opossum and mouse on the other hand. Between frog and Catharrini, finally, there is only a small number of syntenically conserved tDNAs.

We also analyzed the tDNA mobility in two invertebrate clades, drosophilids and nematode genus Caenorhabditis. Within these nematodes, we observe a rather high degree of syntenic conservation, ranging from $45 \%$ between C. elegans and C. japonica up to $84 \%$ for the most closely related pair $C$. remanei and $C$. brenneri. In general, conservation levels are consistent to the known phylogeny of the Caenorhabditis species [47]. For the genus Drosophila with the twelve common representatives, on the other hand, there is much less syntenic conservation. The lowest value is $17 \%$ (D. wilistoni and $D$. persimilis). The best conserved tDNA arrangements are observed between the two closely related species $D$. simulans and D. sechellia with $78 \%$. On average, the percentage of conservation is just around $50 \%$ or less. Full data 
Table 1: Comparison of observed and expected number of tRNA pairs.

\begin{tabular}{|c|c|c|c|}
\hline Species & Observed & Expected & p-value \\
\hline B. taurus & 28452 & 22790 & 0 \\
\hline C. familiaris & 4858 & 4271 & 0 \\
\hline D. novemcinctus & 7918 & 11498 & 1 \\
\hline M. domestica & 7402 & 914 & 0 \\
\hline E. telfairi & 49 & 9.35 & 0 \\
\hline E. caballus & 72 & 4.42 & 0 \\
\hline F. catus & 8792 & 11816 & 1 \\
\hline G. gorilla & 40 & 0.08 & 0 \\
\hline H. sapiens & 97 & 0.27 & 0 \\
\hline L. africana & 1645 & 3553 & 1 \\
\hline M. mulata & 168 & 0.23 & 0 \\
\hline M. murinus & 42 & 0.06 & 0 \\
\hline M. musculus & 1001 & 425 & 0 \\
\hline O. anatinus & 27015 & 25008 & 0 \\
\hline O. lemur & 1364 & 1285 & 0 \\
\hline P. troglodytes & 78 & 0.25 & 0 \\
\hline P.pygmaeus & 83 & 0.28 & 0 \\
\hline O. cuniculus & 118 & 37.15 & 0 \\
\hline R. rattus & 28198 & 16148 & 0 \\
\hline
\end{tabular}

The expectation values are computed by placing the tRNAs at uniformly random position in the genome. Empirical $p$ values are computed from 50 to 1000 replicates.

are shown in Tab. 3 for nematodes and in Additional File 4 for Drosophila.

The sequence conservation of syntenically conserved tRNAs is consistent with the duplication/deletion mechanisms. Additional File 5 shows a neighbor-joining tree of the tRNA-Ala sequences of nematodes, which includes also a few additional species that are not part of the genome-wide survey. We find that syntenically conserved tRNAs genes are typically conserved with an identical sequence across species, even though some tRNAs with the same anticodon located elsewhere in the genome show small sequence variations.

The fraction of syntenically conserved tDNAs correlates with the divergence of the genomes at sequence 
Table 2: Quantity structure of linkage analysis results in vertebrates

\begin{tabular}{|c|c|c|c|c|c|c|c|c|c|c|c|c|c|c|c|c|c|}
\hline \multirow{3}{*}{ Homo Sapiens } & \multirow{4}{*}{493} & \multicolumn{2}{|c|}{ Homo Sapiens } & \multicolumn{2}{|c|}{ Pan Troglodytes } & \multicolumn{2}{|c|}{ Pongo Pygmaeus } & \multicolumn{2}{|c|}{$\begin{array}{l}\text { Macaca } \\
\text { Mulatta }\end{array}$} & \multicolumn{2}{|c|}{ Mus Musculus } & \multicolumn{2}{|c|}{$\begin{array}{l}\text { Monodelphis } \\
\text { Domestica }\end{array}$} & \multicolumn{2}{|c|}{ Gallus Gallus } & \multicolumn{2}{|c|}{$\begin{array}{l}\text { Xenopus } \\
\text { Tropicalis }\end{array}$} \\
\hline & & & & \multicolumn{2}{|c|}{22,312} & \multicolumn{2}{|c|}{20,143} & \multicolumn{2}{|c|}{17,154} & \multicolumn{2}{|c|}{453,512} & \multicolumn{2}{|c|}{167,537} & \multicolumn{2}{|c|}{985} & \multicolumn{2}{|c|}{6341} \\
\hline & & & & 444 & 494 & 438 & 488 & 438 & 387 & 442 & 22,206 & 442 & 20,398 & 366 & 155 & 383 & 332 \\
\hline & & & & 0.9 & 0.98 & 0.89 & 0.95 & 0.89 & 0.86 & 0.9 & 0.91 & 0.9 & 0.94 & 0.74 & 0.93 & 0.78 & 0.57 \\
\hline \multirow[t]{3}{*}{ Pan Troglodytes } & 504 & \multicolumn{2}{|c|}{8,641} & & & \multicolumn{2}{|c|}{22,375} & \multicolumn{2}{|c|}{17,073} & \multicolumn{2}{|c|}{176,153} & \multicolumn{2}{|c|}{182,022} & \multicolumn{2}{|c|}{1,048} & \multicolumn{2}{|c|}{6,201} \\
\hline & & 366 & 394 & & & 497 & 503 & 498 & 391 & 504 & 22,963 & 503 & 20,847 & 400 & 160 & 395 & 364 \\
\hline & & 0.73 & 0.8 & & & 0.99 & 0.98 & 0.99 & 0.87 & 1 & 0.94 & 1 & 0.96 & 0.79 & 0.96 & 0.78 & 0.62 \\
\hline \multirow[t]{3}{*}{ Pongo Pygmaeus } & 512 & \multicolumn{2}{|c|}{8,673} & \multicolumn{2}{|c|}{7,556} & & & & & & & & & 1,0 & & 6,5 & \\
\hline & & 349 & 368 & 330 & 375 & & & 494 & 390 & 512 & 22,716 & 512 & 20,797 & 411 & 158 & 450 & 348 \\
\hline & & 0.68 & 0.75 & 0.64 & 0.74 & & & 0.96 & 0.87 & 1 & 0.93 & 1 & 0.95 & 0.8 & 0.95 & 0.88 & 0.59 \\
\hline Macaca Mulatta & 450 & & & & & & & & & & & & & 9 & & & \\
\hline & & 309 & 368 & 284 & 363 & 286 & 333 & & & 393 & 22,588 & 393 & 20,646 & 332 & 156 & 355 & 347 \\
\hline & & 0.69 & 0.75 & 0.63 & 0.72 & 0.64 & 0.65 & & & 0.87 & 0.93 & 0.87 & 0.95 & 0.74 & 0.94 & 0.79 & 0.59 \\
\hline Mus Musculus & 24352 & & & & & & & & & & & 10,0 & 201 & 65, & & 106 & \\
\hline & & 2,030 & 382 & 2,294 & 369 & 2,126 & 395 & 2,211 & 351 & & & 24,336 & 21,809 & 20,643 & 166 & 20,716 & 422 \\
\hline & & 0.08 & 0.77 & 0.09 & 0.73 & 0.09 & 0.77 & 0.09 & 0.78 & & & 1 & 1 & 0.85 & 1 & 0.85 & 0.72 \\
\hline Monodelphis & 21810 & & & & & & & & & & 815 & & & 67, & & 106 & \\
\hline & & 1,750 & 318 & 2,071 & 363 & 1,846 & 35 & 2,123 & 353 & 16,634 & 19,466 & & & 19,290 & 166 & 19,306 & 416 \\
\hline & & 0.08 & 0.65 & 0.09 & 0.72 & 0.08 & 0.07 & 0.1 & 0.78 & 0.76 & 0.8 & & & 0.88 & 1 & 0.89 & 0.71 \\
\hline Gallus Gallus & 166 & & & & & & & & & & & & & & & 5 & \\
\hline & & 38 & 38 & 38 & 39 & 35 & 38 & 32 & 38 & 132 & 1,169 & 130 & 1276 & & & 142 & 236 \\
\hline & & 0.23 & 0.08 & 0.23 & 0.08 & 0.21 & 0.07 & 0.19 & 0.08 & 0.8 & 0.05 & 0.78 & 0.06 & & & 0.86 & 0.4 \\
\hline Xenopus & 586 & & & & & & & & & & & & & 1 & & & \\
\hline & & 24 & 72 & 25 & 69 & 23 & 65 & 26 & 53 & 126 & 708 & 115 & 630 & 16 & 12 & & \\
\hline & & 0.04 & 0.15 & 0.04 & 0.14 & 0.04 & 0.13 & 0.04 & 0.12 & 0.22 & 0.03 & 0.2 & 0.03 & 0.03 & 0.07 & & \\
\hline
\end{tabular}

The upper right triangle quantifies the single-sided linkage results whereas the lower left triangle represents the number of two-sided linkage analysis results. Each table entry is organized as follows: The top row lists the number of synteny associations; Below, the the sizes of domain and range, i.e., the numbers of tDNAs in the two species, are given. The third line gives the corresponding coverage, i.e., the fraction of syntenically conserved tDNAs in the two species. 
Table 3: Syntenic conservation of tDNAs

\begin{tabular}{lcccccc}
\hline & tDNA & C. briggsae & C. remanei & C. brenneri & C. elegans & C. japonica \\
\hline C. briggsae & 958 & - & 0.84809 & 0.82788 & 0.72691 & 0.68647 \\
C. remanei & 958 & 0.74712 & - & 0.73696 & 0.63603 & 0.55528 \\
C. brenneri & 1587 & 0.61962 & 0.63997 & - & 0.49783 & 0.48763 \\
C. elegans & 820 & 0.77629 & 0.75617 & 0.73602 & - & 0.68558 \\
C.japonica & 1307 & 0.46607 & 0.48633 & 0.49634 & 0.45589 & - \\
\hline
\end{tabular}

The table shows the fraction of tRNA loci between pairs of species. Every field contains the fraction of tDNAs of the species in the column, for which we could find a syntenic position in the row species.

level, Fig. 6 and Tab. 4. The correlation is significant even though the data is rather noisy, a fact that can be explained at least in part by the unavoidable artifacts resulting from our approach. Utilizing annotation data directly to determine local synteny is problematic, for instance, near members of very large recently duplicated gene families. In principle, syntenic conservation could be inferred more accurately from genome-wide alignments. Since tDNAs are treated like repetitive elements in the currently available pipelines, this strategy cannot be employed in practice. Nevertheless, the method provides at least a crude estimate of the tDNA turnover rate, indicating the tDNAs are relocated at time-scales only 25 times slower than background mutation rate, i.e., at an evolutionary distance of 1 mutation per site, $20 \%$ to $60 \%$ of the tDNAs have been deleted or relocated in one lineage.

These values should be regarded as upper bounds of syntenic conservation, i.e., tDNA turnover is probably even faster. For example, the identity of the tDNA (i.e., its anticodon) was not used in the analysis. Despite of the high mobility of tDNAs there are some ancient conserved loci. We further investigated two of the 77 syntenic loci conserved between Xenopus and Human in which tDNAs with the same anticodon were retained. Manual inspection of the flanking protein coding genes confirmed synteny. Neither locus is syntenically conserved in stickleback, lamprey or lancet, however.

\section{Conclusions}

We have developed a pipeline based on tRNAscan$\mathrm{SE}[36]$ to extract and analyze the locations of tRNA genes and pseudogenes of eukaryotic genomes. In our analysis,

Table 4: Parameters of the linear regressions in Fig. 6

\begin{tabular}{lcc}
\hline Clade & $\boldsymbol{\rho}$ & slope \\
\hline Vertebrates & -0.968 & $-0.41 \pm 0.02$ \\
Drosophila & -0.678 & $-0.21 \pm 0.02$ \\
Caenorhabditis & -0.638 & $-0.55 \pm 0.16$ \\
\hline
\end{tabular}

we focus not only on the number of tRNA genes, but also on their relative genomic locations, and in particular on the formation of tDNA clusters. Surprisingly, we found no distinctive clade-specific features or large scale trends, with the exception of the rather straightforward observation that the larger metazoan genomes typically tend to harbour large numbers of tDNAs.

In some species, large clusters of tDNAs occur. This effect has first been reported in Entamoeba histolytica. The origin of this gene organization in the genus Entamoeba clearly predates the common ancestor of the species investigated to date. Their function of the array-like structure remains unclear [32]. We report here that this phenomenon is not restricted to a particular clade of protists but rather appears independently in many times throughout eukaryotes.

In most eukaryotes, tRNAs are multi-copy genes with little or no distinction between paralogs so that orthology is hard to establish, in particular in the presence of tRNA gene clusters. As a consequence, the evolution of genomic tRNA arrangements is non-trivial to study over larger time-scales. Upper bounds on syntenic conservation can be estimated, however, by considering small sets of flanking protein coding genes for which homology information can be retrieved from existing annotation. We found that tRNAs change their genomic location at time-scales comparable to mutation rates: syntenic conservation fades at roughly the same evolutionary distances as sequence conservation in unconstrained regions.

The absence of large numbers of partially degraded tRNA copies in many of the investigated genomes provides a hint at the mechanisms of tRNA mobility: At least in part the relocation events appear to be linked to chromosomal rearrangements rather than mere duplicationdeletion of the tRNA genes themselves. The latter mechanism, which appears to be prevalent e.g. in mitochondrial genomes [48], certainly also plays a role, since tRNA pseudogenes are readily observed in many species, as do tRNA retrogenes [49]. A link between tRNA loci, and in particular tRNA clusters, and chromosomal instability 


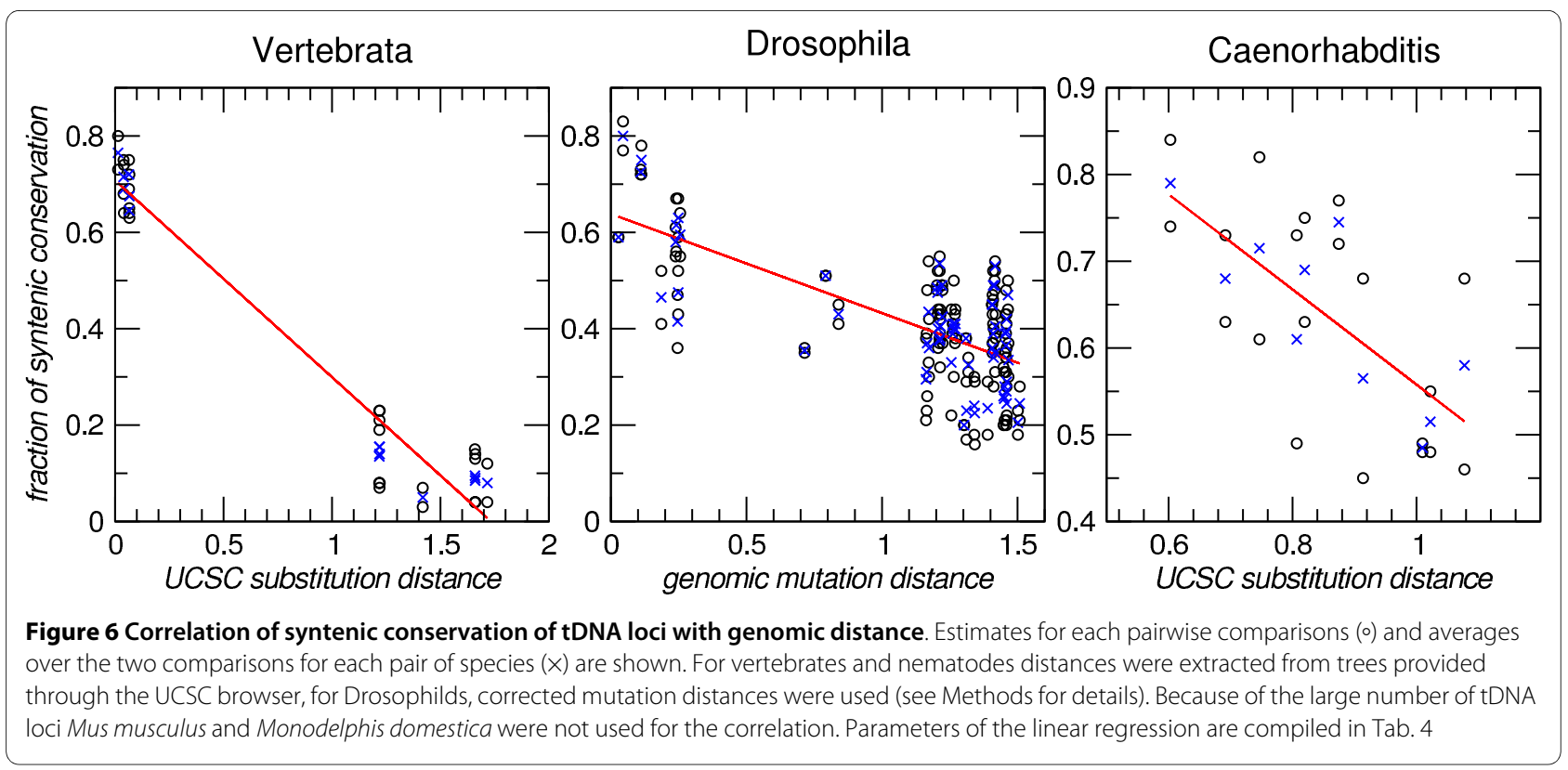

has been pointed out repeatedly in the literature, showing that tRNA genes can interfere with the replication forks [26-30]. The data collected here provide a basis to investigate this connection more systematically in the future.

Overall, the tRNA complement of Eukaryotes is highly dynamic part of the genomes whose organization evolves rapidly and in a highly lineage specific manner - a behavior that is in striking contrast to the extreme conservation of sequence and function of the tRNAs themselves.

\section{Methods}

\section{Sequence data}

We retrieved 74 eukaryotic genome mainly from the following public resources: NCBI, Ensemble Genome Browser and Joint Genome Institute. For a detailed list of the individual genome assemblies we refer to the Additional File 6.

\section{tRNA detection}

Detection of tRNAs was performed by using tRNAscan-SE v.1.23 (April 2002) with default parameters, i.e., the TRNA2 . $\mathrm{cm}$ covariance with strict filter parameter 32.1 was used to screen each genome for tRNAs and tRNA pseudogenes. All analyses were performed using both the set of all intact, putatively functional tRNAs identified by tRNAscan-SE and using all tDNA loci, i.e., the union of tRNA genes and tRNA pseudogenes.

The distinction of tRNA genes and pseudogenes necessarily relies of a set of heuristics implemented in tRNAscan-SE. These are well-founded in what is known about functional tRNA genes [50-55]. Processing and recognition of specific tRNAs imposes stringent constraints on the sequence (and secondary structure) of tRNAs; several nucleotides of mature tRNAs need to chemically modified in most species, imposing further constraints on the primary sequence. tRNAscan-SE's consensus models implement these contraints with reasonable accuracy but by no means perfectly. In the absence of detailed experimental information on the expression and the functionality of a particular tDNA it is of course impossible to distinguish between tRNA genes and tRNA pseudogenes with absolute certainty. For the statistical evaluation of genome-wide comparison reported here, however, the accuracy of tRNAscan-SE appears to be sufficient $[21,36,56]$. There are, however, several sources for errors, in particular in the presence of RNA editing e.g. in the mitochondrial tRNAs of many plants and the protist [57-60]. Such organellar data are not considered in this contribution, however.

tRNA-geo pipeline The tRNA-geo pipeline is a Perl program that parses tRNAscan-SE output and produces summary information as well as overview graphics such as those shown in Figs. 1 and 5. First tDNA locations are sorted in consecutive order along each input sequence, distances are measures (see below for exact definitions), tDNA pairs and tDNA clusters are identified, summary statistics are computed. Graphics are produced using PSTricks macros and LaTeX.

Every tDNA is represented by a quadruple $P=(a, b, o$, $t$ ), where $a<b$ are the start and end positions within each input sequence (chromosome, scaffold, or contig), and $o$ $\in\{+,-\}$ is the orientation of the tRNAs. We say that two tDNAs are of the same type $t$ if they belong the same isoacceptor family, i.e., if they code for the same aminoacid. The tRNA loci are ordered such that $P_{i}<P_{j}$ if and only if $a_{i}<a_{j}$. The distance between two consecutive loci $P_{i}$ and $P_{i+1}$ is defined as $\delta_{i}=a_{i+1}-b_{i}$. 
A cluster $\mathbf{C}$ is a maximal sub-sequence of loci $\mathbf{C}=\left(P_{i}\right.$, $\left.P_{i+1}, \ldots, P_{j}\right)$ such that $\delta_{k}<1000$ for $i \leq k<j$. The cut-off of 1000 was chosen because the overwhelming majority of consecutive tDNA pairs in the random control have larger distances while a large fraction of the tDNA pairs in the real data have smaller distances than this cut-off value, see Fig. 7 here.

A cluster is called homogeneous if all its tDNAs are of the same type $t_{k}$; otherwise, it is called heterogeneous. A sub-sequence consisting of two consecutives loci located within a cluster $\mathbf{C}$ is called a pair. The pair $\left(P_{i}, P_{i+1}\right)$ is homogeneous if $t_{i}=t_{i+1}$ and heterogeneous otherwise. A pair has parallel orientation if $o_{i}=o_{i+1}$. For anti-parallel pairs, $o_{i}=o_{i+1}$, we distinguish head-to-head $o_{i}=+$ and $o_{i+1}$ $=-(\leftarrow \rightarrow)$, and tail-to-tail $o_{i}=-$ and $o_{i+1}=+(\rightarrow \leftarrow)$ orientations.

In order to test whether the observed proportion of homogenous and inhomogeneous pairs depends strongly on whether tRNA pseudogenes are included in the analysis, we used Fisher's exact test. Differences in proportion are significant only in a few species, typically those with very large number of tDNAs (see Additional File 7) suggesting that pseudogenization and degradation of tRNA genes is to a first approximation independent of the mutual positioning of $\mathrm{tDNAs}$.

\section{Simulations}

In order to investigate the statistical significance of the tDNA pairs we compare the genomic tDNA organization with randomized configurations. To this end, we remove the collection of tRNA genes and pseudogenes from the genome and re-insert them at positions chosen from a uniform distribution on the remaining sequence. Empirical $p$-values, defined as

$$
p=\#\{i \mid \gamma(i) \geq x\} / N \text {, }
$$

where $y(i)$ is the number of clustered tRNAs in replicate $i, x$ is the number of clustered tRNAs in the genome, are determined from $N=50$ to $N=1000$ random replicates. For large (insignificant) $p$-values, simulations were terminated at fewer replicates to save computer time.

Statistical tests were performed using the R statistics environment [61]. In particular, Fisher's exact test [62] with $2 \times 2$ contingency tables was used to determine whether different filtering procedures influenced the proportion of homogeneous versus heterogeneous tDNA pairs.

\section{Synteny}

To analyze the synteny between species, we utilized two different pipelines depending on available genomic data and their interrelations in public data sources. The BioFuice [63] integration platform is used to analyze the synteny in eight different vertebrate species Homo sapiens, Pan troglodytes, Pongo pygmaeus, Macaca mulatta, Mus musculus, Monodelphis domestica, Gallus gallus, and Xenopus tropicalis. The analysis runs in several steps. Firstly, the Ensembl data source (version 53) is utilized to create the genomic mappings between the tRNAs and/or tRNA pseudogenes and at most five consecutive proteincoding flanking genes in both directions, up- and downstream. The number 5 was chosen pragmatically as a trade-off between the need to evaluate local information and the unavoidable incompleteness of genome annotations, whence homologs of many genes are missing in individual genomes. These genomic mappings are chromosome- and strand-specific, i.e., the resulting genes are located on the same chromosome and strand as the input tDNAs. Next, the resulting genes are associated to protein-coding genes of other mammalian species using the homologous data available in Ensembl Compara (version

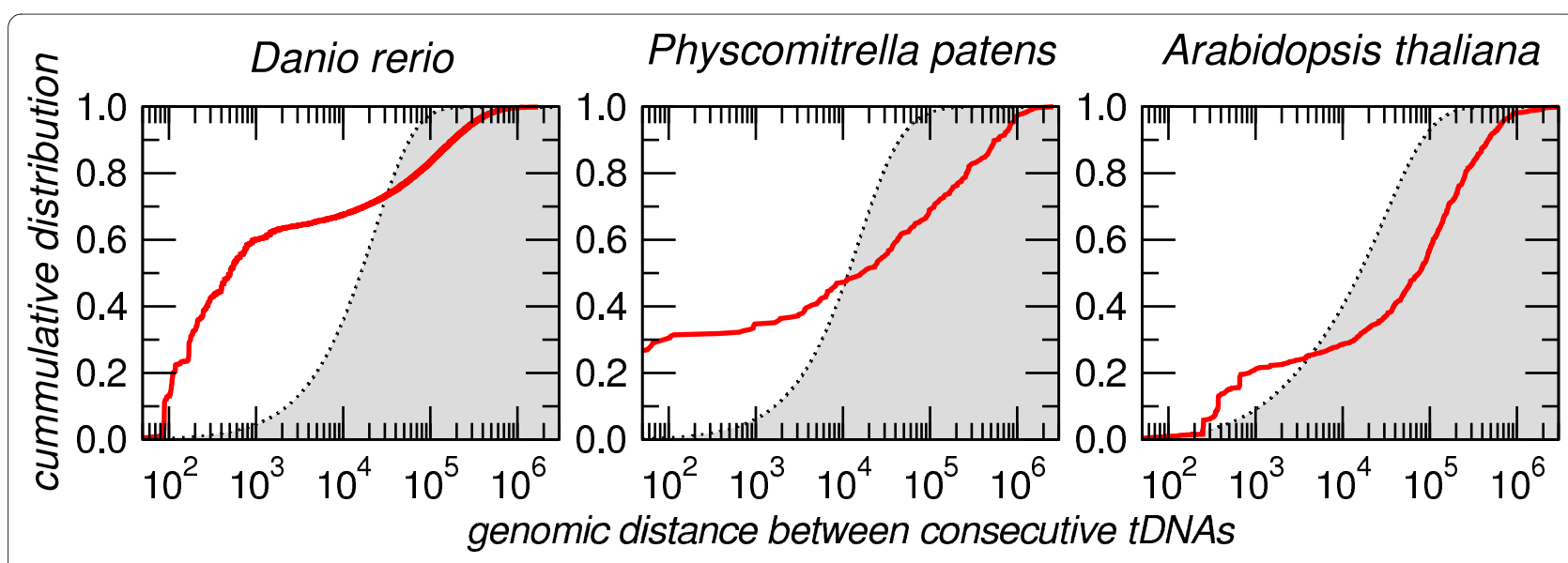

Figure 7 Cumulative distribution of tDNA pairs distances. Measured data are shown in red, random expectation from randomly placed tDNAs are shown as gray background. At a distance of $1000 \mathrm{nt}$ the vast majority of clusters cannot be explained by the random background. 
53). These homology relationships between genes in different species are then filtered to focus on those genes flanking tRNAs. Finally, tDNAs of different mammals can be associated based on the genomic mappings to their flanking genes (gene-tRNA) and the homology relations between those (gene-gene). We consider two alternatives for creating such tDNA relationships:

1. Two tDNAs are associated by the single-sided linkage relation if there is at least one homology relationship between their pre-selected flanking genes. Here we do not require that the homologous genes have the same relative orientation or relative location w.r.t. to the tDNAs.

2. Two tDNAs are associated by the two-sided linkage relation if there is at least one pair of homologous genes in both the up-stream and the down-stream region. Again, relative orientations are not taken into account. The tDNAs need to be located between the two homologous gene-pairs, however.

The Single-sided linkage relation turns out to be not very informative because many-to-many homology relations for large gene families and the relatively large regions used to define the synteny relation severely limit the sensitivity. We therefore limit a details discussion to the two-sided linkage relation.

For invertebrate genomes, synteny information was extracted directly from genome annotation using a custom-made pipeline based on Perl and awk scripts. For the nematodes $C$. elegans, C. briggsae, C. japonica, $C$. brenneri, C. remanei we considered a region of $40.000 \mathrm{nt}$ up- and downstream of the tRNA loci. A pair of tDNAs was defined as syntenic if we could found in this range at least two orthologous proteins between them. The flanking proteins were taken from the genome annotation gfffiles from Wormbase WS204. A list of orthologous proteins was computed using OrthoMCL [64] to determine if two proteins are ortholog. Tab. 3 summarizes the prevalence of tRNA synteny within the genus Caenorhabditis. The tDNAs in the genus Drosophila were analyzed in the same way. The flanking proteins were take from Flybase (release FB2009_09). Since a sufficiently complete orthology annotation was not readily available, we used Proteinortho [65] for this purpose. The results are compiled in Additional File 4.

The fraction of syntenically conserved tDNAs was compared to the evolutionary distances for each pair of genomes in the three data sets described above. The evolutionary distance for the Vertebrates and Nematodes is gathered by the tree model underlying the UCSC 28 -way alignments [66]. For the genus Drosophila the evolutionary distances are genomic mutation distances computed from 4-fold degenerated sites in all coding regions corrected for base composition as in [67].

\section{Additional material}

\begin{abstract}
Additional file 1 Genomic Distribution of tDNAs. Comprehensive overview of the genomic distribution of tRNA genes and tRNA pseudogenes as described in Fig. 1.

Additional file 2 Count of tDNAs and tDNA pair configurations. List of total tDNA predictions including $P$-values and counts of tDNA pair configurations from the empirical data and simulations.

Additional file $\mathbf{3}$ Co-occurrences of tDNAs. Comprehensive summary of co-occurrence data for tDNAs as described in Fig. 5.

Additional file $\mathbf{4}$ Syntenic conservation of Drosophila tDNAs. List of percentage distribution of tDNA synteny in the genus Drosophila.

Additional file $\mathbf{5}$ Sequence conservation of nematode tDNA-Ala Neighbor-joining tree showing that tDNAs usually have identical sequences when they are syntenically conserved, while tRNAs with the same anticodon can exhibit small sequence variations within each species. Additional file 6 Genome versions used in this survey. List of the genome versions and website from which they were downloaded.

Additional file 7 Proportions of tDNA pairs. Summary of the Fisher Test statistic for comparing proportion of pairs configurations.
\end{abstract}

\section{Authors' contributions}

CBS and PFS designed the study, CBS, CSOA, TK, and JE implemented analysis tools and analyzed the data, all authors contributed to the interpretation of the results, CBS and PFS drafted the manuscript, and all authors contributed to, read, and approved the final manuscript.

\section{Acknowledgements}

We thank Borut Jurčič Zlovec (Ljubljana University), Gustavo N. Rubiano (Universidad Nacional de Colombia) for stimulating discussions, four anonymous reviewers for their comments and suggestions, Maribel Hernández-Rosales and Markus Riester for help with Perl programming, and Jens Steuck for technical support. This study would not have been possible without the genome sequence data provided by US Department of Energy Joint Genome Institute, the National Institutes of Health (NIH), NCBI, The Genome Center at Washington University (St. Luis), Broad Institute, The Wellcome Trust Sanger Institute, Baylor College of Human Medicine, Rat Genome project, Agencourt Bioscience, the International Fugu, Medaka, Zebrafish, and Human Genome Consortia, Genoscope, Wormbase, Flybase, J. Craig Venter Institute, PERLEGEN, $T I G R$, and the C. parvum and C. neoformans genome projects. Financial support by the DAAD AleCol program (to CBS), the DFG Bioinformatics Initiative, and the European Community (projects EMBIO, SYNLET, and EDEN) is gratefully acknowledged.

\section{Author Details}

'Bioinformatics Group, Department of Computer Science and Interdisciplinary Center for Bioinformatics, University of Leipzig, Härtelstraße 16-18, D-04107, Leipzig, Germany, ${ }^{2}$ Department of Biology, Universidad Nacional de Colombia. Carrera45 \# 26-85 - Edificio Uriel Gutiérrez, Bogotá D.C., Colombia, ${ }^{3}$ Genedata AG Maulbeerstrasse 46 CH 4016 Basel, Switzerland, 4Biostatistics and Bioinformatics unit, Institute for Research in Biomedicine (IRB Barcelona), Barcelona, Spain, 5 Max Planck Institute for Mathematics in the Sciences, Inselstraß 22 D-04103 Leipzig, Germany, ${ }^{6}$ Fraunhofer Institute for Cell Therapy and Immunology, Perlickstraße 1, D-04103 Leipzig, Germany, ${ }^{7}$ Santa Fe Institute, 1399 Hyde Park Rd, Santa Fe, NM 87501, USA and 8Institute for Theoretical Chemistry, University of Vienna, Währingerstraße 17, A-1090 Wien, Austria

Received: 17 February 2010 Accepted: 28 April 2010

Published: 28 April 2010

\section{References}

1. Gesteland RF, Atkins JF, Eds: The RNA World Plainview, NY: Cold Spring Harbor Laboratory Press; 1993.

2. Eigen $M$, Lindemann BF, Tietze M, Winkler-Oswatitsch R, Dress AWM, von Haeseler A: How old is the genetic code? Statistical geometry of tRNA provides an answer. Science 1989, 244:673-679. 
3. Eigen $M$, Winkler-Oswatitsch R: Transfer-RNA, an early gene? Naturwissenschaften 1981, 68:282-292.

4. Rodin S, S O, Rodin A: Transfer RNAs with complementary anticodons: could they reflect early evolution of discriminative genetic code adaptors? Proc Natl Acad Sci USA 1993, 90:4723-4727.

5. Di Giulio M: The origin of the tRNA molecule: implications for the origin of protein synthesis. J Theor Biol 2004, 226:89-93.

6. Fujishima K, Sugahara J, Tomita M, Kanai A: Sequence evidence in the archaeal genomes that tRNAs emerged through the combination of ancestral genes as 5' and 3' tRNA halves. PLoS One 2008, 3:e1622.

7. Di Giulio M: Formal Proof that the Split Genes of tRNAs of Nanoarchaeum equitans Are an Ancestral Character. J Mol Evol 2009, 69:505-511.

8. Li Y, Luo J, Zhou H, Liao JY, Ma LM, Chen YQ, Qu LH: Stress-induced tRNAderived RNAs: a novel class of small RNAs in the primitive eukaryote Giardia lamblia. Nucleic Acids Res 2008, 36:6048-6055.

9. Shaheen HH, Horetsky RL, Kimball SR, Murthi A, Jefferson LS, Hopper AK: Retrograde nuclear accumulation of cytoplasmic tRNA in rat hepatoma cells in response to amino acid deprivation. Proc Natl Acad Sci USA 2007, 104(21):8845-8850

10. Jöchl C, Rederstorff M, Hertel J, Stadler PF, Hofacker IL, Schrettl M, Haas H, Hüttenhofer A: Small ncRNA transcriptome analysis from Aspergillus fumigatus suggests a novel mechanism for regulation of proteinsynthesis. Nucleic Acids Res 2008, 36:2677-2689.

11. Cole C, Sobala A, Lu C, Thatcher SR, Bowman A, Brown JW, Green PJ, Barton GJ, Hutvagner G: Filtering of deep sequencing data reveals the existence of abundant Dicer-dependent small RNAs derived from tRNAs. RNA 2009, 15:2147-2160.

12. Lee YS, Shibata Y, Malhotra A, Dutta A: A novel class of small RNAs: tRNAderived RNA fragments (tRFs). Genes Dev 2009, 23:2639-2649.

13. Sun FJ, Fleurdépine S, Bousquet-Antonelli C, Caetano-Anollés G, Deragon JM: Common evolutionary trends for SINE RNA structures. Trends Genet 2007, 23:26-33.

14. Rozhdestvensky TS, Kopylov AM, Brosius J, Hüttenhofer A: Neuronal BC1 RNA structure: evolutionary conversion of a tRNA(Ala) domain into an extended stem-loop structure. RNA 2001, 7:722-730.

15. lacoangeli A, Rozhdestvensky TS, Dolzhanskaya N, Tournier B, Schutt J, Brosius J, Denman RB, Khandjian EW, Kindler S, Tiedge H: On BC1 RNA and the fragile $X$ mental retardation protein. Proc Nat/ Acad Sci USA 2008, 105:734-739.

16. Nishihara H, Smit A, F ON: Functional noncoding sequences derived from SINEs in the mammalian genome. Genome Res 2006, 16:864-874.

17. Frenkel FE, Chaley MB, Korotkov EV, Skryabin KG: Evolution of tRNA-like sequences and genome variability. Gene 2004, 335:57-71.

18. Withers M, Wernisch L, dos Reis M: Archaeology and evolution of transfer RNA genes in the Escherichia coli genome. RNA 2006, 12:933-942.

19. Copeland CS, Marz M, Rose D, Hertel J, Brindley PJ, Bermudez Santana C, Kehr S, Stephan-Otto Attolini C, Stadler PF: Homology-based annotation of non-coding RNAs in the genomes of Schistosoma mansoni and Schistosoma japonicum. BMC Genomics 2009, 10:464

20. Higgs PG, Jameson D, Jow H, Rattray M: The evolution of tRNA-Leu genes in animal mitochondrial genomes. J Mol Evol 2003:435-445.

21. Goodenbour JM, Pan T: Diversity of tRNA genes in eukaryotes. Nucleic Acids Res 2006, 34:6137-6146.

22. Marck C, Grosjean H: tRNomics: analysis of tRNA genes from 50 genomes of Eukarya, Archaea, and Bacteria reveals anticodon-sparing strategies and domain-specific features. RNA 2002, 8:1189-1232.

23. Marck C, Kachouri-Lafond R, Lafontaine I, Westhof E, Dujon B, Grosjean H The RNA polymerase III-dependent family of genes in hemiascomycetes: comparative RNomics, decoding strategies, transcription and evolutionary implications. Nucleic Acids Res 2006, 34:1816-1835

24. McFarlane RJ, Whitehall SK: tRNA genes in eukaryotic genome organization and reorganization. Cell Cycle 2009, 8:3102-3106

25. Talbert PB, Henikoff S: Chromatin-based transcriptional punctuation. Genes Dev 2009, 23:1037-1041.

26. Di Rienzi S, Collingwood D, Raghuraman MK, Brewer B: Fragile genomic sites are associated with origins of replication. Genome Biol Evol 2009, 2009:350-363.

27. Labib K, Hodgson B: Replication fork barriers: pausing for a break or stalling for time? Embo reports 2007, 8:8492-8501.
28. Admire A, Shanks L, Danz N, Wang M, Weier U, Stevens W, Hunt E, Weinert $\mathrm{T}$ : Cycles of chromosome instability are associated with a fragile site and are increased by defects in DNA replication and checkpoint controls in yeast. Genes \& Dev 2006, 20:159-173.

29. Deshpande AM, Newlon CS: DNA Replication Fork Pause Sites Dependent on Transcription. Science 1996, 272:1030-1033.

30. Ivessa AS, Lenzmeier BA, Bessler JB, Goudsouzian LK, Schnakenberg SL, Zakian VA: The Saccharomyces cerevisiae helicase Rrm3p facilitates replication past nonhistone protein-DNA complexes. Mol Cell 2003, 12:1525-1536

31. Sugahara J, Kikuta K, Fujishima K, Yachie N, Tomita M, Kanai A: Comprehensive analysis of archaeal tRNA genes reveals rapid increase of tRNA introns in the order thermoproteales. Mol Biol Evol 2008, 25:2709-2716

32. Tawari B, Ali IK, Scott C, Quail MA, Berriman M, Hall N, Clark CG: Patterns of evolution in the unique tRNA gene arrays of the genus Entamoeba. Mol Biol Evol 2008, 25:187-198.

33. Clark CG, Ali IK, Zaki M, Loftus BJ, Hall N: Unique organisation of tRNA genes in Entamoeba histolytica. Mol Biochem Parasitol 2006, 146:24-29.

34. Molnár A, Schwach F, Studholme DJ, Thuenemann EC, Baulcombe DC: miRNAs control gene expression in the single-cell alga Chlamydomonas reinhardtii. Nature 2007, 447:1126-1129.

35. Zhao T, Li G, Mi S, Li S, Hannon G, Wang X, Qi Y: A complex system of small RNAs in the unicellular green alga Chlamydomonas reinhardtii. Genes Dev 2007, 21:1190-1203.

36. Lowe TM, Eddy SR: tRNAscan-SE: a program for improved detection of transfer RNA genes in genomic sequence. Nucleic Acids Res 1997, 25:955-964

37. Supplementary Data in Machine-Readable Form 2009 [http:// www.bioinf.uni-leipzig.de/Publications/SUPPLEMENTS/09-050].

38. Kuo C, Ochman H: Deletional Bias across the Three Domains of Life. Genome Biol Evol 2009, 2009:145-52.

39. Rocha EPC: Codon usage bias from tRNA's point of view: Redundancy, specialization, and efficient decoding for translation optimization. Genomes Res 2004, 14:2279-2286.

40. Higgs $P$, Ran W: Coevolution of codon usage and tRNA genes leads to alternative stable states of biased codon usage. Mol Biol Evol 2008 25:2279-2291.

41. Mukhopadhyay P, Basak S, Ghosh TC: Nature of selective constraints on synonymous codon usage of rice differs in GC-poor and GC-rich genes. Gene 2007, 400:71-81.

42. Nozawa M, Kawahara Y, Nei M: Genomic drift and copy number variation of sensory receptor genes in humans. Proc Natl Acad Sci USA 2007, 104:20421-20426.

43. Perry GH, Yang F, Marques-Bonet T, Murphy C, Fitzgerald T, Lee AS, Hyland C, Stone AC, Hurles ME, Tyler-Smith C, Eichler EE, Carter NP, Lee C, Redon $R$ : Copy number variation and evolution in humans and chimpanzees. Genome Res 2008, 18:1698-1710.

44. Chung T, Siol O, Dingermann T, Winckler T: Protein interactions involved in tRNA gene-specific integration of Dictyostelium discoideum nonlong terminal repeat retrotransposon TRE5-A. Mol Cell Biol 2007 , 27:8492-8501.

45. Gordon J, Byrne K, Wolfe K: Additions, losses, and rearrangements on the evolutionary route from a reconstructed ancestor to the modern Saccharomyces cerevisiae genome. PLoS Genet 2009, 5:e1000485.

46. Wei F, Li S, Ma HR: Computer simulation of tRNA evolution. J Phys A Math Theor 2009, 42:345101

47. Kiontke K, Fitch DHA: The phylogenetic relationships of Caenorhabditis and other rhabditids. Wormbook 2005 [http://www.wormbook.org]. C elegans Research Communit T, Wormbook Doi/10.1895/wormbook.1.11.1

48. Rawlings TA, Collins TM, Bieler R: Changing identities: tRNA duplication and remolding within animal mitochondrial genomes. Proc Acad Natl USA 2003, 100:15700-15705

49. Volff JN, Brosius J: Modern genomes with retro-look: retrotransposed elements, retroposition and the origin of new genes. Genome Dyn 2007, 3:175-190

50. Hou YM, Schimmel P: A simple structural feature is a major determinant of the identity of a transfer RNA. Nature 1988, 333:140-145.

51. de Duve C: Transfer RNA: the second genetic code. Nature 1988, 333:117-118. 
52. Schimmel P, Giegé R, Moras D, Yokoyama S: An operational RNA code for amino acids and possible relationship to genetic code. Proc Nat/ Acad Sci USA 1993, 90:8763-8768.

53. Hohn MJ, Park HS, O'Donoghue P, Schnitzbauer M, Söll D: Emergence of the universal genetic code imprinted in an RNA record. Proc Natl Acad Sci USA 2006, 103:18095-18100.

54. Goto-Ito S, Ito T, Kuratani M, Bessho Y, Yokoyama S: Tertiary structure checkpoint at anticodon loop modification in tRNA functional maturation. Nat Struct Mol Biol 2009, 16:1109-1115.

55. Pütz J, Giegé R, Florentz C: Diversity and similarity in the tRNA world: overall view and case study on malaria-related tRNAs. FEBS Lett 2010, 584:350-358.

56. Abe T, Ikemura T, Ohara Y, Uehara H, Kinouchi M, Kanaya S, Yamada Y, Muto A, Inokuchi H: tRNADB-CE: tRNA gene database curated manually by experts. Nucleic Acids Res 2009, 37:D163-D168.

57. Lonergan KM, Gray MV: Editing of transfer RNAs in Acanthamoeba castellanii mitochondria. Science 1993, 259:812-816.

58. Marechal-Drouard L, Kumar R, Remacle C, Small I: RNA editing of larch mitochondrial tRNA(His) precursors is a prerequisite for processing. Nucleic Acids Res 1996, 24:3229-3234.

59. Fey J, Weil J, Tomita K, Cosset A, Dietrich A, Small I, Marechal-Drouard L: Role of editing in plant mitochondrial transfer RNAs. Gene 2002, 286:21-24.

60. Leigh J, Lang BF: Mitochondrial 3' tRNA editing in the jakobid Seculamonas ecuadoriensis: a novel mechanism and implications for tRNA processing. RNA 2004, 10:615-621.

61. The R Project for Statistical Computing [http://www.r-project.org/]

62. Fisher RA: On the interpretation of $\chi^{2}$ from contingency tables, and the calculation of $P$. J Royal Stat Soc 1922, 85:87-94.

63. Kirsten T, Rahm E: BioFuice: Mapping-based data intergation in bioinformatics. Data Integration in the Life Sciences, of Lect Notes Comp Sci 2006, 4075:124-135. Third International Workshop, DILS 2006

64. Li L, Stoeckert CJ Jr, Roos DSR: OrthoMCL: Identification of Ortholog Groups for Eukaryotic Genomes. Genome Res 2003, 13:2178-2189.

65. Lechner M, Steiner L, Prohaska SJ: Proteinortho - Orthology detection tool. 2009 [http://www.bioinf.uni-leipzig.de/Software/proteinortho/].

66. Miller W, Rosenbloom K, Hardison RC, Hou M, Taylor J, Raney B, Burhans R, King DC, Baertsch R, Blankenberg D, Kosakovsky Pond SL, Nekrutenko A, Giardine B, Harris RS, Tyekucheva S, Diekhans M, Pringle TH, Murphy WJ, Lesk A, Weinstock GM, Lindblad-Toh K, Gibbs RA, Lander ES, Siepel A, Haussler D, Kent WJ: 28-way vertebrate alignment and conservation track in the UCSC Genome Browser. Genome Res 2007, 17:1797-1808.

67. Tamura K, Subramanian S, Kumar S: Temporal patterns of fruit fly (Drosophila) evolution revealed by mutation clocks. Mol Biol Evol 2004, 21:36-44.

doi: 10.1186/1471-2164-11-270

Cite this article as: Bermudez-Santana et al., Genomic organization of eukaryotic tRNAs BMC Genomics 2010, 11:270

\section{Submit your next manuscript to BioMed Central} and take full advantage of:

- Convenient online submission

- Thorough peer review

- No space constraints or color figure charges

- Immediate publication on acceptance

- Inclusion in PubMed, CAS, Scopus and Google Scholar

- Research which is freely available for redistribution

Submit your manuscript at www.biomedcentral.com/submit
C Biomed Central 